\title{
Transport of Bone in Femur over an Intramedullary Nail: A Novel Corticotomy Technique
}

\author{
Palanivelayutham Sivakumar ${ }^{1}$, Ramesh Perumal ${ }^{2}$, Sudipta K Patra ${ }^{3}$, Dheenadhayalan Jayamaraju ${ }^{4}$, \\ Shanmuganathan Rajasekaran ${ }^{5}$
}

\begin{abstract}
Introduction: Despite successful union after intramedullary nailing of open femur fractures with bone loss or significant comminution, the discrepancy in leg length can lead to a significant impairment for the patient. Lengthening over a femoral nail is a described technique in such clinical scenarios. Corticotomy around an existing intramedullary nail (IMN) was introduced to address lengthening of the limbs without changing or removing any previously implanted IMN. Osteotomy around an existing IMN is a challenging technique that requires careful handling to avoid inadvertent damage to the IMN.

Case description: Two patients with open type IIIA shaft of femur fractures who presented with bone loss following trauma underwent debridement and IMN fixation. After nailing, the patient was placed on a fluoroscopic table to enable an anteroposterior and lateral radiograph of the femur. Under image guidance, a rail frame was applied and we used a novel technique of osteotomy for lengthening of the femur using a Gigli saw. In both cases, the bony union was achieved by the end of 1 year.

Conclusion: Lengthening over the IMN using a lengthening device helps in correcting limb length discrepancy as well as aids in achieving optimal bony union at the same time. The use of our novel corticotomy technique helps to minimize the damage to the intramedullary device. The existence of the nail minimizes the time required for the external frame since during the consolidation phase the nail supports the regenerate bone.

Keywords: Bone loss in femur, Corticotomy, Gigli saw, Intramedullary nail, Limb length discrepancy, Posttraumatic deformity, Transport over nail. Journal of Orthopedics and Joint Surgery (2021): 10.5005/jp-journals-10079-1031
\end{abstract}

\section{INTRODUCTION}

The treatment of fractures with bone loss remains a challenge. However, with the recent advancements in soft tissue reconstruction, limbs previously considered for amputation are being salvaged. Reconstruction of extremities in the presence of significant bone loss usually involves multiple surgeries which not only is timeconsuming but also has a significant psychological impact on the patient. The traditional options to treat bone loss with bone grafting (vascularized fibula/iliac crest transfers), induced membrane technique and bone transport techniques, although useful are technically demanding surgeries. Bone transport techniques can use either plate fixation, intramedullary nail (IMN) fixation, or external fixator application. Among these available options, bone transport over an IMN has the following advantages-locked nail maintains length and alignment, better patient compliance due to shortened ex-fix time, accurate docking, and early mobilization of joints. We report a novel technique of osteotomy for lengthening the femur over an existing IMN.

\section{Surgical Technique}

The patient with bone loss was first stabilized with an IMN based on the length determined by the opposite side femur by preoperative planning (Fig. 1). After nailing, the patient was placed on a fluoroscopic table to enable an anteroposterior and lateral radiograph of the femur. Preoperative planning was done to calculate the length of the femur using the unaffected side femur length. The external fixator frame is then inserted parallel to the IMN. Care is taken to avoid contact between the internal

\author{
${ }^{1-4}$ Department of Orthopaedics and Trauma, Ganga Hospital, \\ Coimbatore, Tamil Nadu, India \\ ${ }^{5}$ Department of Orthopaedics, Ganga Hospital, Coimbatore, Tamil
} Nadu, India

Corresponding Author: Palanivelayutham Sivakumar, Department of Orthopaedics and Trauma, Ganga Hospital, Coimbatore, Tamil Nadu, India, Phone: +91 9994465917, e-mail: drspsiva@gmail.com

How to cite this article: Sivakumar P, Perumal R, Patra SK, et al. Transport of Bone in Femur over an Intramedullary Nail: A Novel Corticotomy Technique. J Orth Joint Surg 2021;3(1):54-57.

Source of support: Nil

Conflict of interest: None

and external fixation. The external frame is applied using two pins coated with hydroxyapatite in the proximal and distal segments (Fig. 2A). The osteotomy site is placed in the metaphyseal bone and at a site different from the original fracture. The osteotomy is performed through an incision of $7 \mathrm{~cm}$, along the lateral aspect of the thigh with the site marked under fluoroscopy assistance. Through the lateral incision, the anterolateral cortex is cut with the help of a $\mathrm{k}$ wire in a drill mount and the anteromedial, medial, posteromedial cortex is cut with the help of Gigli saw to complete the osteotomy (Figs 2B to F). Care is taken to prevent damage to the IMN. Using fluoroscopy and tactile spatial sensation, the osteotomy is performed. The purpose is to achieve corticotomy by approaching the bone from the periphery by avoiding the intramedullary unit. To ensure that the osteotomy is complete, 

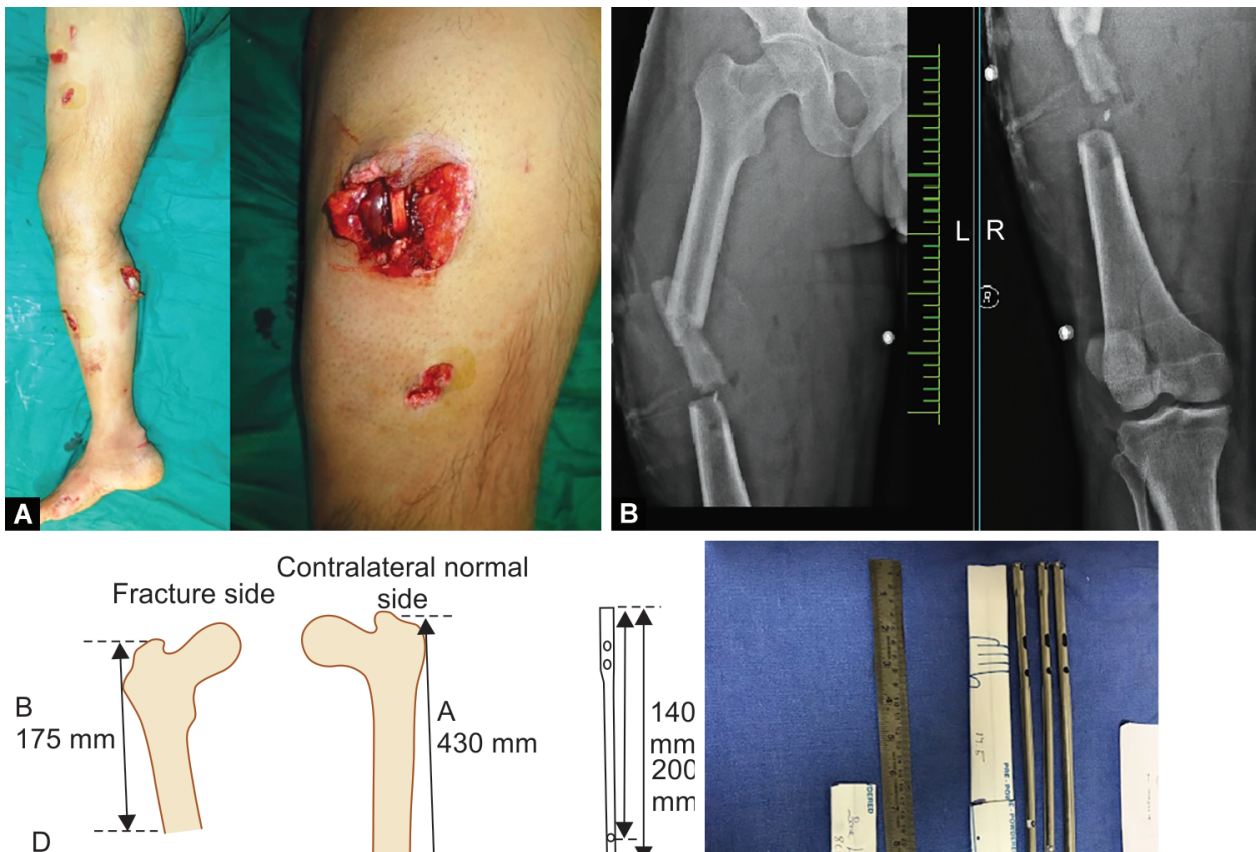

Contralateral normal

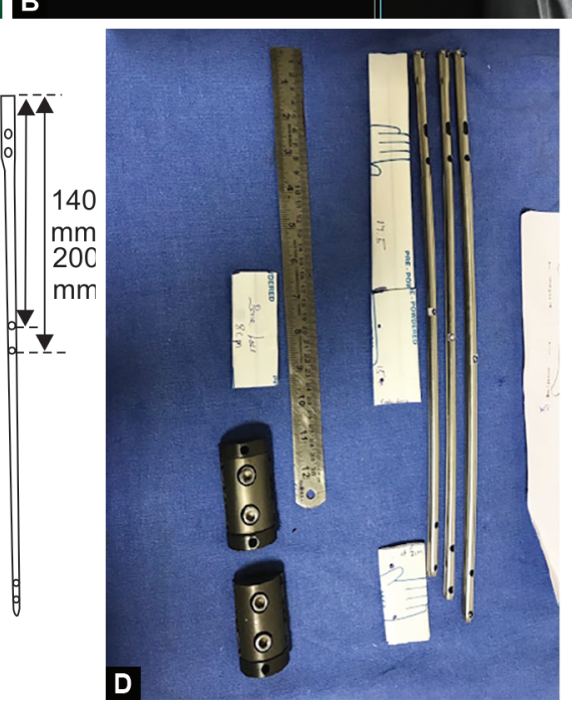

\section{C}

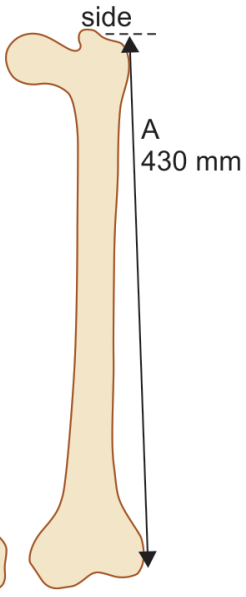

Figs $1 A$ to D: (A) Open injury picture of the patient; (B) Preoperative radiograph showing comminuted segmental shaft of femur fracture; (C) Preoperative planning measuring the bone loss; (D) Customized nail with extra holes to hold the transport unit and prevent collapse

a manual distraction component of the compression-distraction device is used. Distraction is started on postoperative day 11 at a rate of $1 \mathrm{~mm}$ per day. Serial follow-up radiographs are taken to confirm distraction at the osteotomy site.

\section{Case Examples}

Two patients with Gustillo Anderson Type III-A open shaft of femur fracture presented to us with bone loss (Case example 1 and Case example 2) (Fig. 3). The patient underwent initial debridement and fixation with an IMN maintaining the length. Under image guidance, a rail frame was applied. Through a lateral incision, the lateral aspect of the osteotomy site was exposed. With the help of $\mathrm{k}$ wire and Gigli saw, osteotomy was completed in a sequential manner using our above-mentioned technique. In both the patients, distraction was started from the 11th postoperative day and the fracture went to unite by the end of 1-year follow-up (Fig. 3).

\section{Ethics Approval and Informed Consent}

The research procedure was reviewed and approved by the institutional review board, and informed consent was obtained.

\section{Discussion}

Limb length discrepancies are very commonly seen in clinical practice. Although small discrepancies are tolerable and usually asymptomatic, greater discrepancies contribute to substantial disability. ${ }^{1}$ Bone lengthening is a common procedure performed with an external fixator or IMN to treat the discrepancy of the limb length, but complications like axial deformities, contractures, and adjacent joint stiffness can occur in the presence of an external fixator. ${ }^{2}$ These complications were reduced by performing femoral lengthening over an IMN which gives good biomechanical stability to allow bony healing. Reconstruction with osteotomy around an existing nail with a lengthening device seems to be an effective procedure for limb length discrepancy. ${ }^{3,4}$ It provides support to the regenerate bone and decreases the duration in which the patient can return to normal activities. In this technique, the osteotomy was done using $\mathrm{k}$ wire and a Gigli saw without much blood loss. The technique of using an osteotome during the osteotomy can cause inadvertent damage to the IMN. ${ }^{5}$ Hence, the Gigli saw-a novel osteotomy technique-gives us a very safe alternative. The nail used in case example 1 was customized during preoperative planning in which two extra holes were made in mediolateral direction at 190 and $200 \mathrm{~mm}$ length to pass extra screws to hold the bone transport in position to prevent any collapse during weight-bearing. During the consolidation phase, the nail ensured the stability of the structure and thus the external fixator was removed easily following the distraction stage. ${ }^{6}$ The limitation of the study is a higher risk of infection because of the presence of an external 

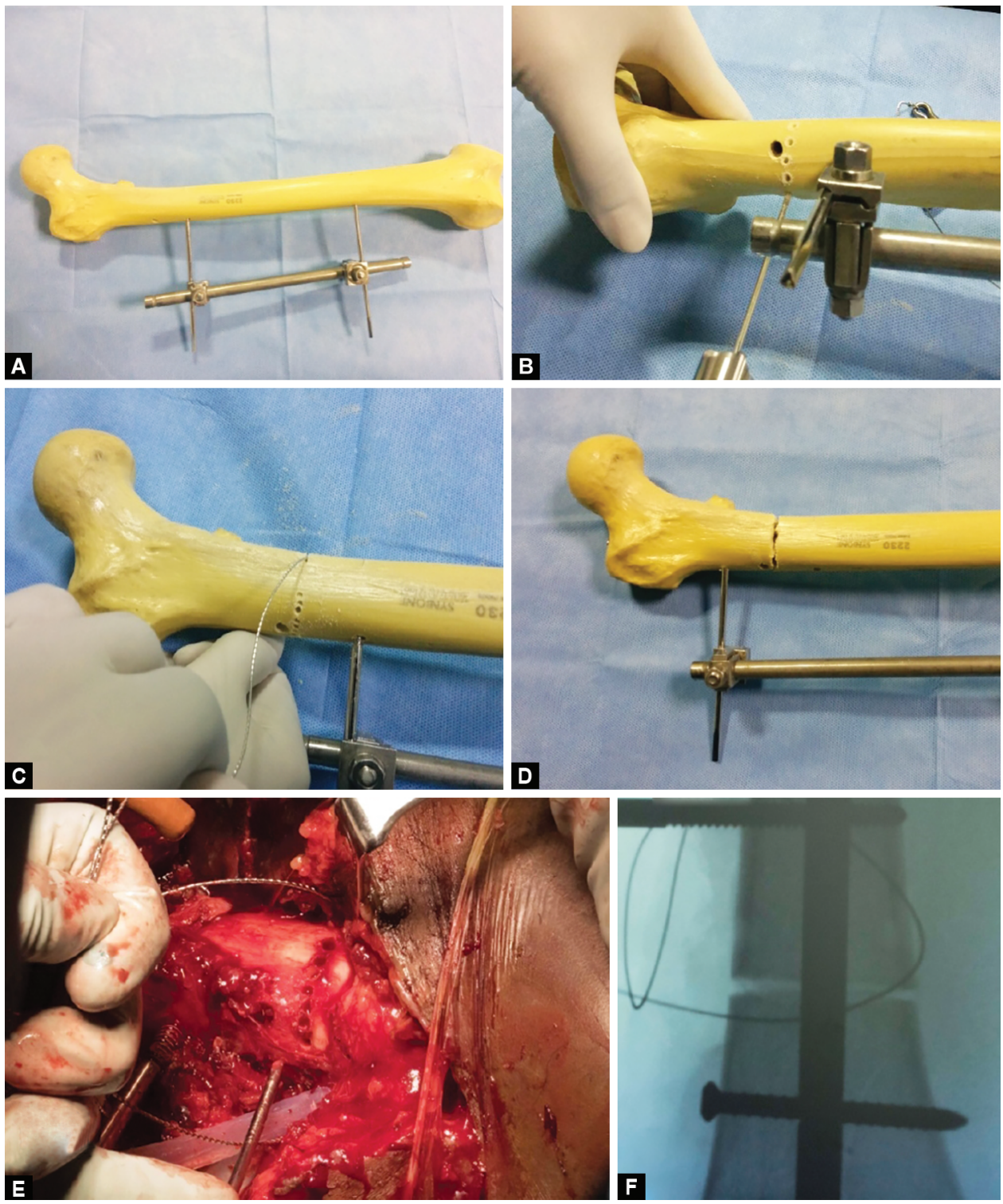

Figs $2 \mathrm{~A}$ to $\mathrm{F}$ : (A) Osteotomy explained in a bone model with femur on an external frame; (B) Lateral cortex multiple drills made with $\mathrm{K}$ wire; (C) Anteromedial, medial and posteromedial cortices are cut with Gigli saw; (D) Completion of osteotomy; (E) Clinical picture of osteotomy being performed using $\mathrm{k}$ wire to perform drill holes in near cortex and Gigli saw to complete the osteotomy in far cortex without any damage to the IMN nail; (F) Radiograph while performing osteotomy

fixator as any superficial pin tract infection may spread along with the IMN. To minimize this possible complication, it is important to educate the patients regarding signs of pin tract infections and early intervention can avoid this complication. In this study, we used HA-coated pins to provide better frame stability, reduced loosening, and lower pin infection rates. ${ }^{7}$ Intramedullary lengthening nails, such as the mechanical intramedullary skeletal kinetic distractor, and the newer motorized nails, such as the Fit bone and Precice, have been recently created. ${ }^{8}$ However, some authors report these devices to be very expensive and are reported to have complications like implant failure due to malfunctioning remote controllers. ${ }^{9}$ These devices still need longer follow-up studies for definitive evaluation.

\section{Conclusion}

This novel osteotomy technique using Gigli saw is a simpler technique that can be easily reproduced, causes less damage to the
IMN with minimal blood loss, and ensures the desired result in an optimal time frame. Lengthening over the IMN using a lengthening device helps in correcting limb length discrepancy as well as aids in achieving optimal bony union at the same time. The use of the Gigli saw for corticotomy helps to minimize the damage to the intramedullary device. The existence of the nail minimizes the time required for the external frame since during the consolidation phase the nail supports the regenerate bone. This novel technique decreases the duration of immobilization of the patient and helps in early return to work.

\section{Clinical Message}

Lengthening over an IMN still provides a valid option for the treatment of lower limb discrepancy, particularly in clinical scenarios of open fractures with bone loss. This novel osteotomy technique is easily reproducible, causes less damage to the IMN, and ensures optimal results within the required time frame. 

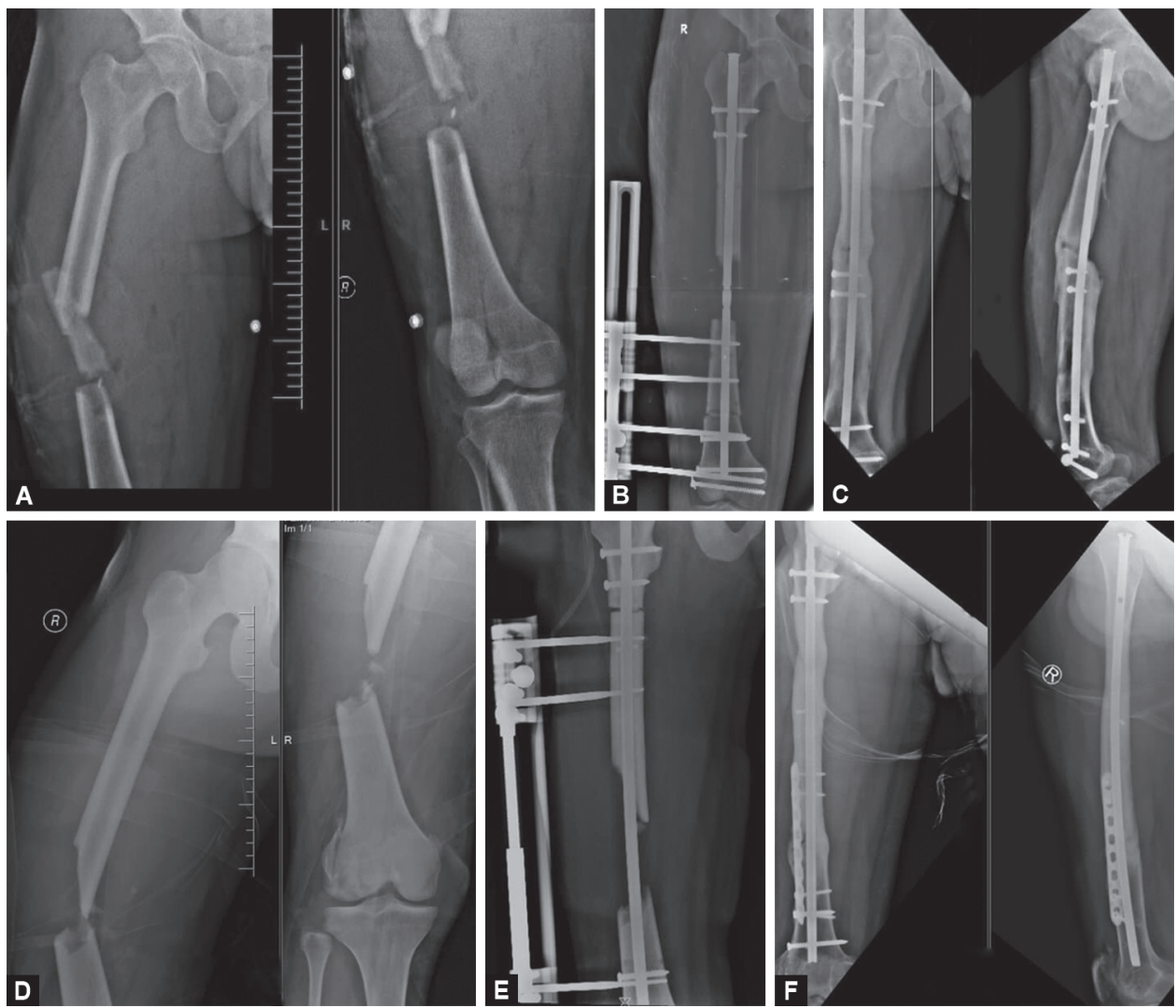

Figs 3A to F: (A) Preoperative radiograph of segmental femur fracture in case example 1; (B) Post-osteotomy radiograph of case example 1; (C) Final follow-up showing united shaft of femur fracture with implant in situ in case example 1 ; (D) Preoperative radiograph of comminuted shaft of femur fracture in case example 2; (E) Post-osteotomy radiograph of case example 2; (F) Final follow-up showing united shaft of femur fracture with implant in situ in case example 2

\section{References}

1. Harris I, Hatfield A, Walton J. Assessing leg length discrepancy after femoral fracture: clinical examination or computed tomography? ANZ J Surg 2005;75(5):319-321. DOI: 10.1111/j.1445-2197.2005.03349.x.

2. Guidera KJ, Hess WF, Highhouse KP, et al. Extremity lengthening: results and complications with the Orthofix system. J Pediatr Orthop 1991;11(1):90-94. DOI: 10.1097/01241398-199101000-00017.

3. Emara KM, Mahran MA, Ghaly NAM, et al. Comparison between lengthening over nail and conventional llizarov lengthening: a prospective randomized clinical study. Strategies Trauma Limb Reconstr 2013;8(2):97-101. DOI: 10.1007/s11751-013-0163-x.

4. Guo Q, Zhang T, Zheng Y, et al. Tibial lengthening over an IMNin patients with short stature or leg-length discrepancy: a comparative study. Int Orthop 2012;36(1):179-184. DOI: 10.1007/s00264-011-1246-2.

5. Boutsiadis A, losifidou E, Nikolaos X, et al. Lengthening over an existing intramedullary nail in cases of post-traumatic femoral shortening. Technical note. Case series study. The Open Orthopaed J 2016;10(1):12. DOI: 10.2174/1874325001610010012.

6. Chaudhary M. Limb lengthening over a nail can safely reduce the duration of external fixation. Indian J Orthopaed 2008;42(3):323. DOI: 10.4103/0019-5413.41857.

7. Pizà G, Caja VL, González-Viejo MA, et al. Hydroxyapatite-coated external-fixation pins. The effect on pin loosening and pin-track infection in leg lengthening for short stature. J Bone Joint Surg $\mathrm{Br}$ 2004;86(6):892-897. DOI: 10.1302/0301-620X.86B6.13875.

8. Cole JD, Justin D, Kasparis T, et al. The intramedullary skeletal kinetic distractor (ISKD): first clinical results of a new IMN for lengthening of the femur and tibia. Injury 2001;32(Suppl 4):SD129-SD139. DOI: 10.1016/S0020-1383(01)00116-4.

9. Accadbled F, Pailhé R, Cavaignac $E$, et al. Bone lengthening using the Fitbone $\left({ }^{\oplus}\right)$ motorized intramedullary nail: the first experience in France. Orthop Traumatol Surg Res 2016;102(2):217-222. DOI: 10.1016/j.otsr.2015.10.011. 